'Unidad de Nutrición Pública. Instituto de Nutrición y Tecnología de los Alimentos, INTA, Universidad de Chile. Santiago, Chile.

Trabajo no recibió financiamiento. Los autores declaran no tener conflictos de interés.

Recibido el 12 de marzo de 2019, aceptado el 8 de agosto de 2019 .

Correspondencia a: Dr. Fernando Vio del Río Unidad de Nutrición Pública,

Instituto de Nutrición y Tecnología de los Alimentos, INTA. El Líbano 5524, Macul, Santiago, Chile. fvio@inta.uchile.cl

\section{Descripción de la progresión de la obesidad y enfermedades relacionadas en Chile}

\author{
FERNANDO VIO $^{1}$, JULIANA KAIN ${ }^{1}$
}

\section{Increasing frequency of obesity in Chile}

Background: An explosive increase in overweight and obesity occurred in Chile since the 90's, without an integrative national policy. Aim: To describe the current obesity situation in Chile and its consequences. Material and Methods: Analysis of the available anthropometric data obtained by an annual census of anthropometry for students (Nutritional Map) collected by the Ministry of Education, and three National Health Surveys (2003, 2009-2010 y 20162017) performed by the Ministry of Health in adults. Results: According to the Nutritional Map the prevalence of obesity in 1987 was $7.5 \%$ among children of six years of age and increased to 24\% in 2018 ( $8 \%$ with severe obesity). At 14 years of age, the frequency of obesity increased from 8 to 15\% from 2011 to 2018. In children older than 15 years, overweight and obesity increased from 61 to $74 \%$ from 2003 to 2016. In the same period, total obesity increased from 23 to $34 \%$ and the frequency of diabetes duplicated from 6 to $12 \%$. The frequency of hypertension did not change, but its prevalence is 45 and $73 \%$ after 45 and 65 years of age, respectively. Conclusions: The frequency of obesity will continue to increase, producing severe health risks for the population. Public policies addressing both structural and individual aspects are recommended.

(Rev Med Chile 2019; 147: 1114-1121)

Key words: Nutrition Status; Nutritional Surveys; Obesity; Overweight.

\section{C}

hile pasó de una situación de pre transición epidemiológica en la década del 70 a una de post transición a fines de los 80 . En los años 60 y 70 la desnutrición era un problema grave, la que fue disminuyendo progresivamente hasta la crisis económica de 1982-1986, que incrementó nuevamente el déficit nutricional ${ }^{1}$. En esa época prácticamente no había obesidad infantil, pero si en adultos, especialmente en mujeres de nivel socioeconómico bajo $^{2}$, coexistiendo la desnutrición con obesidad, lo que se denomina "doble carga de enfermedad"3.

En la década del 90, la situación cambió rápidamente con la erradicación de la desnutrición como problema de salud pública ${ }^{4}$ y al mismo tiempo se produjo un incremento de la obesidad debido principalmente al aumento en el consumo de alimentos procesados, con alto contenido de grasas, azúcar y sal ${ }^{5}$ y la aparición de cadenas de comida rápida con el modelo de "dieta occidental" o "western diet"6. Junto a lo anterior, aumentó el sedentarismo por la compra de televisores y vehículos, junto a un mayor tiempo de permanencia en el trabajo (promedio 10 horas diarias en la Región Metropolitana de Chile), lo que sumado el tiempo de traslado de 2 a 3 horas diarias, no dejaba tiempo libre para cocinar ni hacer actividad física ${ }^{7}$.

Frente al incremento de la obesidad han existido diferentes programas para enfrentarla desde la alimentación y actividad física, como VIDA Chile (1998-2005), Estrategia Global contra la Obesidad (2006-2010) y Elige Vivir Sano (2011-2014), pero 
sin una política integrada y continua en el tiempo que permita detener la obesidad ${ }^{8}$, que ha seguido aumentando. El propósito de este artículo es describir el aumento sostenido en la prevalencia de obesidad en población infantil y adulta y sus consecuencias, con el fin de recomendar políticas públicas para enfrentarla.

\section{Metodología}

Este artículo se basa en instrumentos de instituciones gubernamentales cuyo objetivo es recolectar datos sobre el estado nutricional de la población infantil y adulta y enfermedades crónicas relacionadas. Estos instrumentos son el Mapa Nutricional a cargo de La Junta Nacional de Auxilio Escolar y Becas (JUNAEB) del Ministerio de Educación y las Encuestas Nacionales de Salud (ENS) del Ministerio de Salud.

\section{A. Prevalencia de obesidad infantil y adolescente}

\section{Mapa nutricional}

Estudio censal que dimensiona el estado nutricional (desnutrición, normal, sobrepeso y obesidad) de preescolares, estudiantes de primer y quinto grado de educación básica y primer año de educación media (en promedio de 4 a 15 años). Los datos se recolectan en cada escuela del país y luego se envían a la JUNAEB mediante una plataforma web para posteriormente realizar el procesamiento y análisis de la información.

En la comparación de los resultados del estado nutricional de estudiantes de primero y quinto año básico a través del tiempo es necesario señalar que el 2007 se cambió la referencia para clasificar el estado nutricional NCHS a la de OMS $2007^{9}$ que considera como punto de corte el indicador IMC con su desviación estándar (DE). Un estudio realizado por Atalah y cols. el año $2012^{10}$ comparó ambas referencias para establecer si había diferencias significativas en el estado nutricional, concluyendo que la clasificación a los 6 y 14 años de edad no mostraba diferencias importantes.

Desde el año 2017 se agregó la obesidad severa para aquellos niños que tienen más de $3 \mathrm{DE}$ de la mediana, de acuerdo a la Referencia OMS $2007^{9}$ y el año 2018 se agregó por primera vez la medición de quinto año básico ${ }^{11}$.

\section{B. Prevalencia de obesidad en adultos}

\section{Encuesta Nacional de Salud (ENS)}

La ENS que depende del Ministerio de Salud es una encuesta poblacional de tipo transversal, cuya población objetivo son personas mayores de 15 años chilenas o extranjeras que residen habitualmente en viviendas particulares ocupadas y localizadas en zonas urbanas y rurales de las quince regiones de Chile. Estas encuestas tienen representatividad nacional, regional y urbano/rural. La encuesta incluye datos provenientes de una entrevista personal en el hogar, con un sistema de captura electrónica mediante Tablet aplicada por encuestador y profesional enfermera de acuerdo al tipo de cuestionario ${ }^{12}$.

Para la evaluación del estado nutricional se utilizó el Índice de Masa Corporal (IMC) de acuerdo a OMS. Se calculó IMC en $\mathrm{kg} / \mathrm{m}^{2}$ y se clasificó el estado nutricional según la norma de la OMS en bajo peso $(<18,5)$, normopeso $(18,5$ a $24,9)$, sobrepeso $(25$ a 29,9$)$, obesidad $(30-39,9)$ y obesidad mórbida $(\geq 40)^{13}$.

Análisis Estadístico: se verificó si existen diferencias significativas entre las cifras de obesidad de los adolescentes en los 3 años analizados que reporta el Mapa Nutricional y las prevalencia de obesidad y diabetes observadas en las tres ENS en 2 fases: a) comparando los 3 años simultáneamente utilizando el test ANOVA para muestras independientes y b) comparando 2 años a la vez, utilizando el test de Bonferroni. Se consideró como significativo un $\mathrm{p}<0,05$.

\section{Resultados}

\section{Obesidad en niños y adolescentes}

Los datos de primero básico que entrega el Mapa Nutricional desde 1987 muestran que la prevalencia de obesidad fue de 7,5\% en 1987 y de 24,4\% el año 2018 (Figura 1). En adolescentes de primer año medio, la obesidad siempre se había mantenido baja con $8,2 \%$ el 2011, pero se incrementó a $13,4 \%$ el 2016 y a $14,7 \%$ el 2018 , resultando estadísticamente significativa la diferencia entre el año 2011 y los años 2016 y 2018 . No hubo diferencia significativa entre 2016 y 2018 (Figura 2). El año 2018 se midió por primera vez la obesidad en quinto año básico, resultando una prevalencia de $27,7 \%$, mayor que en primero básico. 


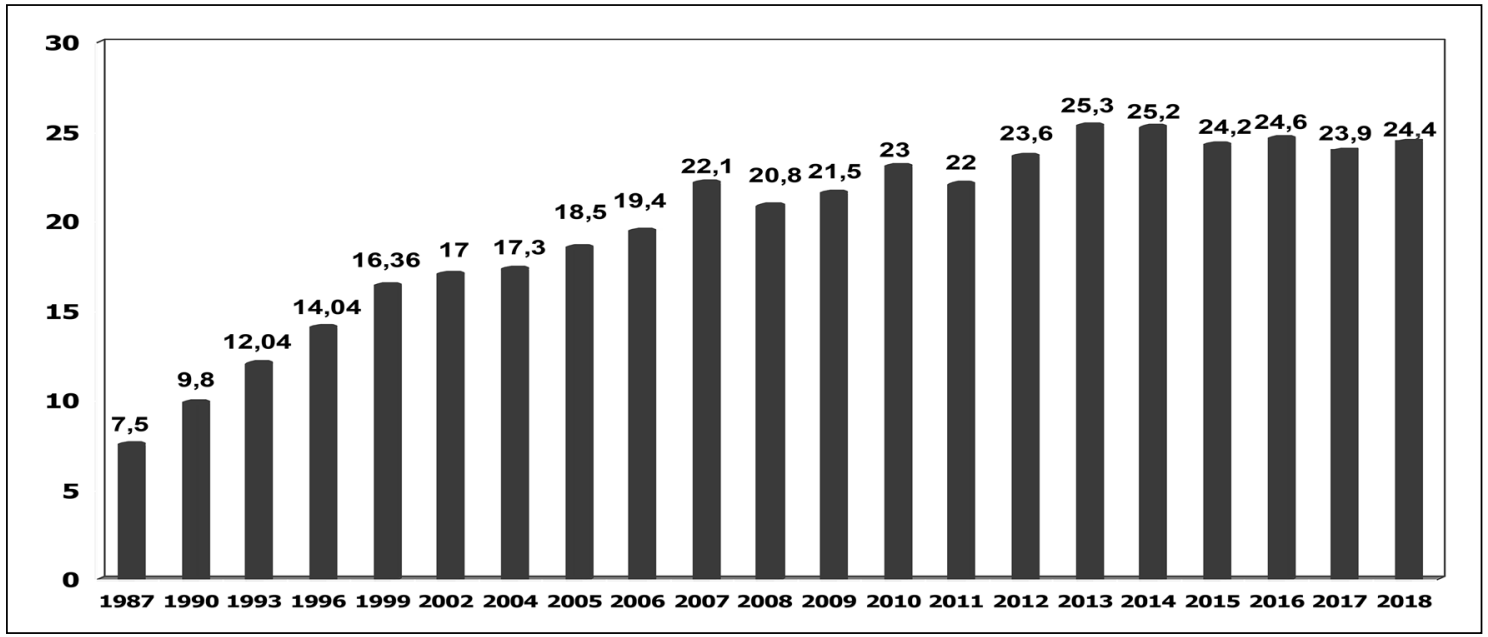

Figura 1. Serie temporal de la prevalencia de obesidad en escolares de primer año básico (6 años) 1987-2018 (en \%). Fuente: Mapa Nutricional (JUNAEB).

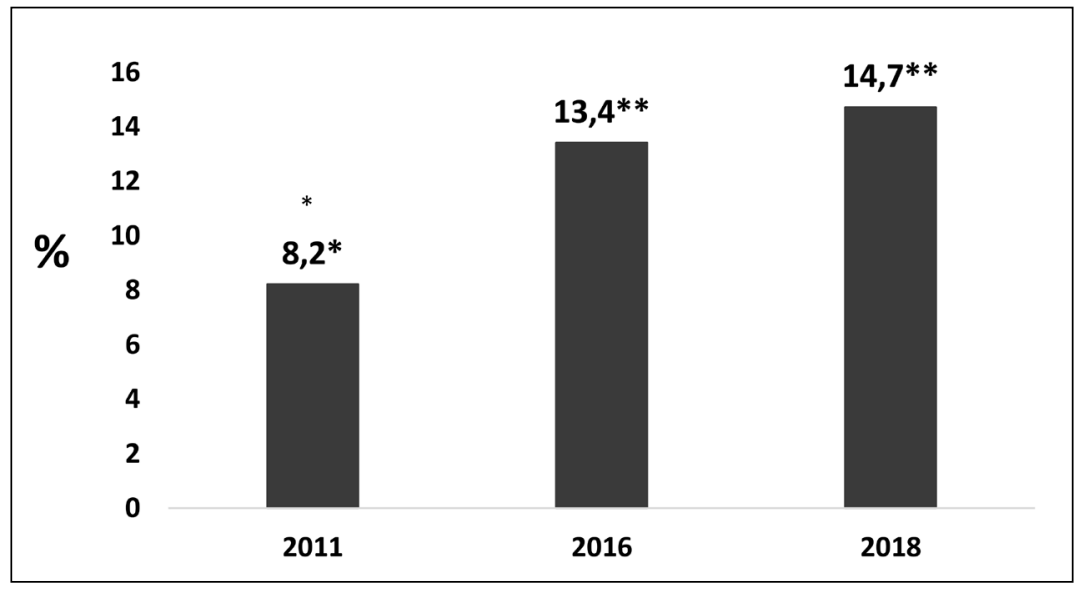

Figura 2. Prevalencia de obesidad en escolares de primero medio (14 años) 2011-2016- 2018 (en $\%)$. ${ }^{*} p<0,001$. Diferencia significativa 2011 con 2016 y 2018. **No hay diferencia significativa entre 2016 y 2018. Fuente: Mapa Nutricional (JUNAEB).
Los primeros datos de obesidad severa $(+3 \mathrm{DE}$ por sobre la mediana) del año 2017 muestran que $8,1 \%$ de los alumnos de primer año básico tiene obesidad severa (10,3\% en los hombres y $5,8 \%$ en las mujeres), lo cual significa que un tercio de los niños obesos tiene problemas graves, con alta posibilidad de tener hipertensión arterial, diabetes e hiperlipidemias. En primero medio, la obesidad severa fue de $2,1 \%$. Estos datos no variaron el 2018, donde la obesidad severa fue de $8 \%$ en prekinder, $8,3 \%$ en kínder, $8,1 \%$ en primero básico, $4,8 \%$ en quinto básico y $1,9 \%$ en primero medio.

\section{Obesidad en adultos}

Las Encuestas Nacionales de Salud (ENS) de los años 2003, 2010-2011 y 2016-2017 han permitido conocer el estado nutricional de la población mayor de 15 años a partir de tres cortes transversales (Figura 3). La ENS 2003 mostró una prevalencia de $21,9 \%$ de obesidad, $1,3 \%$ de obesidad mórbida y $37,8 \%$ de sobrepeso, o sea $61 \%$ de la población con sobrepeso y obesidad. La ENS 2009-2010 mostró un aumento de la obesidad a 25,1\% y del sobrepeso a $39,3 \%$ y la obesidad mórbida se duplicó a 2,3\%, llegando a $66,7 \%$ de la población con sobrepeso y obesidad. 


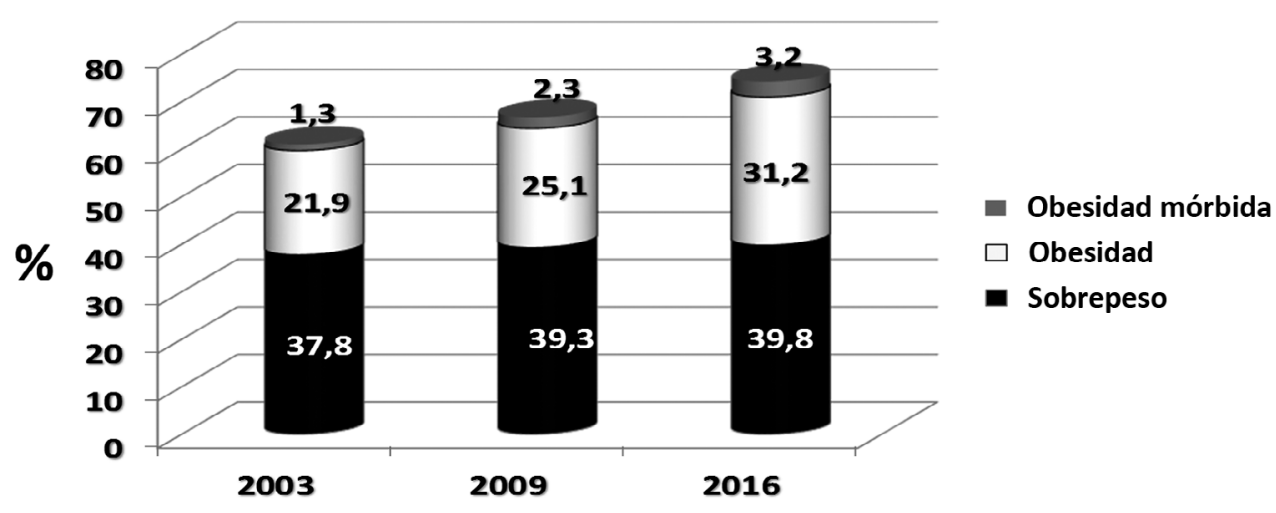

Figura 3. Prevalencia de sobrepeso, obesidad y obesidad mórbida en mayores de 15 años en tres mediciones transversales: 2003, 2009-2010 y 2016-2017 (en\%). Fuente: Encuestas Nacionales de Salud 2003, 2009-2010 y 2016-2017 (MINSAL).

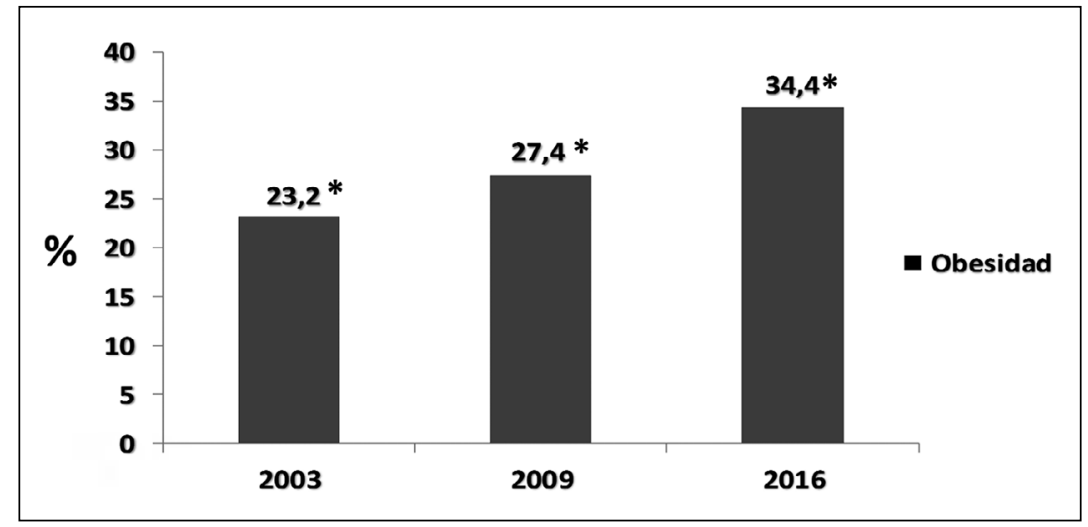

Figura 4. Prevalencia de obesidad total en mayores de 15 años en Chile en tres mediciones transversales: 2003, 2009-2010y 2016-2017 (en\%). ${ }^{*} p<0,0001$. Diferencia significativa en los tres años simultáneamente y además entre 2003 con 2009 y 2016 y 2009 con 2016. Fuente: Encuestas Nacionales de Salud 2003, 2009-2010 y 2016-2017 (MINSAL).
La ENS 2016-2017 mostró un incremento de la obesidad aún mayor que el esperado, llegando a $74,2 \%$ de la población. Lo grave es que este incremento fue en obesidad total, ya que el sobrepeso se mantuvo estable, cercano a $40 \%$. Sin embargo, la obesidad, incluyendo la obesidad mórbida, aumentó de 23,2\% el 2003 a 34,4\% el 2016, siendo esta diferencia estadísticamente significativa entre los tres años comparados (2003, 2009-2010 y 2016-2017) (Figura 4). En la última ENS la obesidad fue mayor en las mujeres $(39,4 \%)$ que en hombres $(30,3 \%)$ y mayor en el nivel socioeconómico bajo, medido por años de escolaridad menor a 8 años $(40,2 \%)$ que en el alto, con más de 12 años de escolaridad (29,5\%). Además, la obesidad se incrementa con la edad, siendo de $40,2 \%$ en el grupo de 30 a 49 años y de $41,7 \%$ en el grupo de 50 a 64 años. Llama la atención que en los mayores de 65 años la obesidad se mantiene elevada $(35,6 \%)$.

\section{Co-morbilidades de la obesidad: Diabetes e hipertensión arterial}

Como consecuencia directa del incremento de la obesidad en Chile, la diabetes tipo 2 se duplicó en 13 años, de 6,3\% el 2003, 9,4\% el 2009-2010 a $12,4 \%$ el 2016-2017, siendo esta diferencia significativa entre las tres encuestas (Figura 5). En cambio, la hipertensión arterial se ha mantenido estable cercana a $27 \%$, pero con un importante incremento después de los 45 años de edad, en que aumenta a $45,1 \%$ y especialmente después de los 65 años, en que la prevalencia es de 73,3\% (Figura 6). 

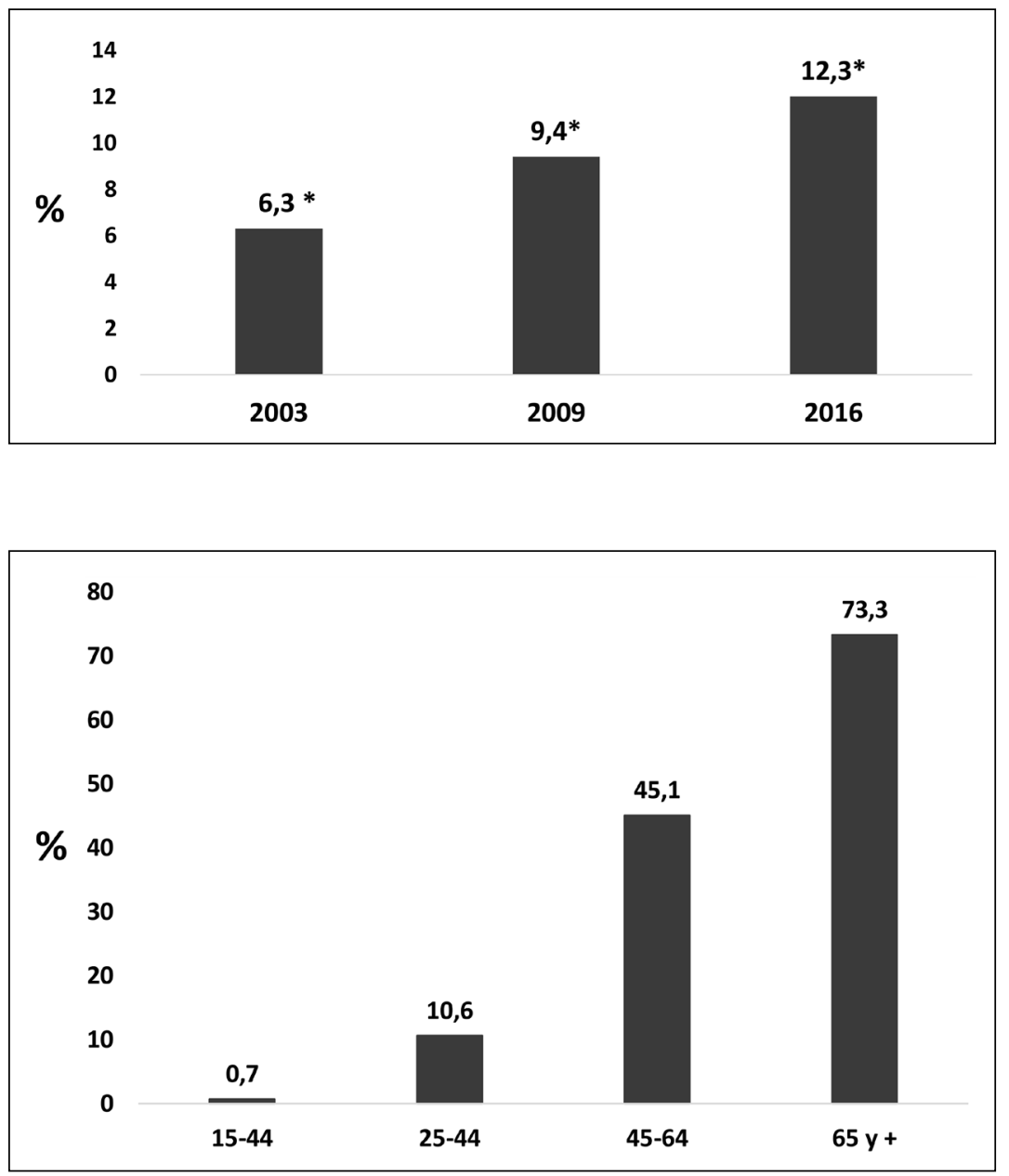

Figura 5. Prevalencia de diabetes mellitus tipo 2 en mayores de 15 años en tres mediciones transversales: 2003, 2009-2010 y 2016-2017 (en\%). *p $<0,0001$. Diferencia significativa en los tres años simultáneamente y además entre 2003 con 2009 y 2016 y 2009 con 2016. Fuente: Encuestas Nacionales de Salud 2003, 2006-2007 y 2016-2017 (MINSAL).
Figura 6. Hipertensión arterial según grupos de edad (20162017). Prevalencia (\%). Fuente: Encuesta Nacional de Salud 2016-2017 (MINSAL).

\section{Discusión}

Los principales hallazgos de este estudio muestran que en Chile el sobrepeso y obesidad están aumentando en forma alarmante. En los niños de 6 años la prevalencia se triplicó en las últimas tres décadas; en los adolescentes de 14 años, aumentó en $60 \%$ en los últimos 7 años, mientras que en adultos aumentó $60 \%$ entre el 2003 y 2017, duplicándose en ese período la prevalencia de diabetes.

Es muy preocupante que la obesidad infantil siga aumentando en todos los grupos de edad, ya que la mayor parte de estos niños continuarán siendo obesos en el futuro ${ }^{14}$. Esto es de gran trascendencia para el país, ya que entre las primeras causas de consulta y hospitalización en Chile se encuentran la hipertensión arterial, diabetes y obesidad, patologías directamente relacionadas con alimentación y sedentarismo ${ }^{15}$.

De acuerdo a publicaciones recientes, nuestro país está en el décimo lugar de países con mayor prevalencia de obesidad infantil en el mundo, lista encabezada por Arabia Saudita, Egipto, Estados Unidos y México en el sexto lugar ${ }^{16}$.

También en el resto de los países la obesidad continúa aumentando. En el año 2015 existían 107,7 millones de niños y 603,7 millones de adultos con obesidad a nivel mundial. Desde 1980, la obesidad se duplicó en más de 70 países ${ }^{17}$ y un tercio de la población tiene obesidad o sobrepeso. De acuerdo a la última información disponible de Estados Unidos, la prevalencia de obesidad en ese país aumentó de 30,5\% en 1999-2000 a 39,6\% el 
2015-2016 ${ }^{18}$, incremento semejante al de Chile que fue de $23,2 \%$ el 2003 a 34,4\% el 2016-2017 (Figura 4).

El aumento de la prevalencia de la obesidad en el mundo podría explicar que las muertes por enfermedades cardiovasculares no están disminuyendo en Australia, Austria, Brasil, Alemania, Holanda, Gran Bretaña y USA ${ }^{19}$. Además, existe preocupación de que la mortalidad pueda estar aumentando por primera vez desde $1970^{20}$.

Las consecuencias de la obesidad incluyen las enfermedades cardiovasculares, cánceres, demencia, asma, enfermedades hepatobiliares, diabetes y enfermedades renales ${ }^{21}$. La prevalencia de obesidad continúa aumentando en todos los países del mundo, con más de 1 millón de muertes debidas a diabetes 2 , medio millón a enfermedades renales relacionadas con diabetes y 180.000 a enfermedad del hígado graso no alcohólico (NAFLD, por sus siglas en inglés) y esteatohepatitis no alcohólica (NASH), que es una forma de hígado graso no alcohólico en la que además de acumulación de grasa en el hígado hay inflamación y lesiones de células hepáticas, que se relaciona con cáncer de hígado y cirrosis. La esteatohepatitis no alcohólica (NASH) se debe a la resistencia a insulina derivada de la obesidad y puede estar presente en $10 \%$ a $35 \%$ de la población adulta global $^{22}$.

El crecimiento económico ha llevado a un aumento del consumo en general y de alimentación en particular, lo cual ha sido estimulado por las empresas de alimentos que han incrementado la variedad de productos procesados y ultra-procesados con alto contenido de grasas, azúcar y sal, los cuales son muy atractivos y apetitosos, lo que incide en el aumento de su consumo ${ }^{23}$. Esto ha ido acompañado de una publicidad sumamente atractiva que llega a todos los hogares para aumentar sus ventas y ganancias, aun cuando sea en detrimento de la salud de las personas ${ }^{24}$.

La Organización Mundial de la Salud (OMS) plantea que para enfrentar la obesidad en el plano individual se requiere limitar la ingesta energética procedente de grasa total y de azúcares; aumentar el consumo de frutas y verduras, así como de legumbres, cereales integrales y frutos secos, y realizar una actividad física periódica de 60 min diarios para los niños y adolescentes y 150 min semanales de actividad moderada o 75 min semanales de actividad vigorosa para los adultos $^{25,26}$.
Para enfrentar la obesidad existen propuestas costo efectivas, como las señaladas por Margozzini et al. ${ }^{27}$ de prolongar la lactancia materna, reformular alimentos procesados, etiquetar los alimentos de acuerdo a la Ley $20.606^{28}$, implementar un sistema de impuestos a alimentos con exceso de grasa, azúcar y sal y entregar subsidios a alimentos saludables como frutas y verduras ${ }^{29}$. Además, se plantea regular la publicidad, monitorear y evaluar las intervenciones y promover la actividad física. Todas estas propuestas son razonables y pueden implementarse en los países, pero requieren de voluntad política que no está siempre presente.

A estas propuestas deben agregarse intervenciones educativas de alimentación y nutrición en establecimientos educacionales, mejorando el entorno educacional con un mejor acceso a alimentos saludables y prohibiendo la venta de los que no son saludables, junto con incorporar la educación en el currículum y en las actividades extracurriculares ${ }^{30}$. Aun cuando estas intervenciones educacionales no han demostrado ser tan costo-efectivas como las anteriores por tener resultados en el largo plazo, son las únicas que en el futuro pueden cambiar los hábitos en la población.

\section{Conclusiones}

Para detener el aumento de la obesidad, la evidencia muestra que lo más costo efectivo en el corto plazo es aplicar medidas estructurales como el impuesto sobre bebidas azucaradas, subsidios a los alimentos saludables y reducir el contenido de grasa, azúcar y sal de los alimentos procesados, junto con garantizar la disponibilidad de alimentos saludables como frutas y verduras, creando "entornos saludables" en establecimientos educacionales $^{30}$. Sin embargo, si estas medidas se aplican sin entregar contenidos sobre alimentación saludable ni fomentando la actividad física desde las edades más tempranas, no se lograrán resultados en el largo plazo. Por esta razón se requieren políticas públicas y legislación que obligue al Ministerio de Educación a incorporar estos temas en forma prioritaria en las actividades curriculares y extracurriculares de los establecimientos educacionales, como está estipulado en los artículos cuarto y sexto de la Ley $20.606^{28}$. 


\section{Referencias}

1. Vio F, Kain J, Gray E. Nutritional Surveillance: The case of Chile. Nutr Res 1992; 12: 321-5.

2. Albala C, Vio F, Olivares S, Andrade M. Efecto de un programa de control de la obesidad en mujeres de nivel socio-económico bajo. Rev Med Chile 1986; 114: 934-8.

3. Kain J, Vio F, Albala C. Childhood nutrition in Chile: from déficit to excess. Nutr Res 1998; 18: 1825-37.

4. Uauy R, Albala C, Kain J. Obesity trends in Latin America: transiting from under to overweight. J Nutr 2001; 131: 893S-899S.

5. Vio F, Albala C. Nutrition Policy in the Chilean Transition. Public Health Nutr 2000; 3: 49-55.

6. Popkin BM. The Nutrition Transition and Obesity in the Developing World. J Nutr 2001; 131: 871S-873S.

7. Albala C, Vio F, Kain J, Uauy R. Nutrition transition in Chile: determinants and consequences. Public Health Nutr 2002; 123-8.

8. Vio F, Albala C, Kain J. Nutrition transition in Chile revisited: mid-term evaluation of obesity goals for the period 2000-2010. Public Health Nutr 2008; 11 (4): 405-12.

9. World Health Organization (WHO). The WHO 2007 SAS Macro Package. World Health Organization (WHO): Ginebra, Suiza. 2007.

10. Atalah E, Loaiza S, Taibo M. Estado nutricional en escolares chilenos según la referencia NCHS y OMS 2007. Nutr Hosp 2012; 27: 1-6.

11. Junta Nacional de Auxilio Escolar y Becas (JUNAEB), Elige Vivir Sano, Contrapeso. Mapa Nutricional 2018. Resumen Estado Nutricional. Disponible en: https:// www.junaeb.cl/wpcontent/uploads/2013/03/MapaNutricional2018_Junaeb_21.02.pdf (Consultado el 26 de febrero de 2019).

12. Ministerio de Salud de Chile, 2017. Encuestas Nacionales de Salud. Disponible en https://www.minsal.cl/ wp-content/uploads/2017/11/ENS-2016-17 (Consultado el 10 enero de 2019)

13. World Health Organization (WHO), Body Mass Index (BMI). Disponible en: http://www.euro.who.int/en/ health-topics/disease-prevention/nutrition/a-healthy-lifestyle/body-mass-index-bmi (Consultado el 19 enero de 2019).

14. Simmonds M, Llewellyn A, Owen CG, Woolacott N. Predicting adult obesity from childhood obesity: a systematic review and meta-analysis. Obes Rev 2016; 17: 95-107.

15. MINSAL, Anuario Consultas y Hospitalizaciones. Departamento de Estadísticas e Información de Salud, DEIS Ministerio de Salud. Disponible en: http://www.
deis.cl/wp-content/uploads/2015/04/IBS-2014.pdf. (Consultado el 19 enero de 2019).

16. NCD Risk Factor Collaboration (NCD-RisC) (2017) Worldwide trends in body-mass index, underweight, overweight, and obesity from 1975 to 2016: a pooled analysis of 2416 population-based measurement studies in 128.9 million children, adolescents, and adults. Lancet 2017; 390: 2627-54.

17. GDB 2015 Obesity Collaborators. Health effects of overweight and obesity in 195 countries over 25 years. NEJM 2017; 377: 13-27.

18. Hales CM, Carroll MD, Fryar CD, Ogden CL. Prevalence of obesity among adults and youth: United States, 2015-2016. NCHS Data Brief 2017; 288: 1-8.

19. Roth GA, Nguyen G, Forouzanfar MH, Mokdad AH, Naghavi M, Murray CJ. Estimates of global and regional premature cardiovascular mortality in 2025. Circulation 2015; 132: 1270-82.

20. Swinburn BA, Sacks G, Hall KD, McPherson K, Finegood DT, Moodie ML, et al. The global obesity pandemic: shaped by global drivers and local environments. Lancet 2011; 378: 804-14.

21. GBD 2017 Risk Factor Collaborators. Global, regional, and national comparative risk assessment of 84 behavioral, environmental and occupational, and metabolic risks or clusters of risks for 195 countries and territories, 1990-2017: a systematic analysis for the Global Burden of Disease Study 2017. Lancet 2018; 392: 1923-94.

22. Younossi Z, Anstee QM, Marietti M, Hardy T, Henry L, Eslam M, et al. Global burden of NAFLD and NASH: trends, predictions, risk factors and prevention. Nat Rev Gastroenterol Hepatol 2018; 15: 11-20.

23. Cediel G, Reyes M, da Costa Louzada ML, Martínez Steele E, Monteiro CA, Corvalán C, et al. Ultra-processed foods and added sugars in the Chilean diet (2010). Public Health Nutr 2018; 21: 125-33.

24. Olivares S, Zacarías I, González CG. Motivaciones y barreras de los niños chilenos; amenazas y oportunidades para la implementación de las Guías Alimentarias Chilenas del 2013. Nutr Hosp 2014; 30: 260-6.

25. OMS. Obesidad y sobrepeso, 16 de febrero de 2018. Disponible en: http://www.who.int/es/news-room/factsheets/detail/obesity-and-overweight. (Consultado el 20 enero de 2019).

26. WHO. Global Strategy on Diet, Physical Activity and Health. https://www.who.int/dietphysicalactivity/factsheet_adults/en/. (Consultado el 29 de julio de 2019).

27. Margozzini P, Bambs C. Obesidad: Un problema de salud colectiva que requiere soluciones colectivas. En: Mardones F, Arnaiz P, Barja S, Rosso P., eds. Obesidad en Chile ¿Qué podemos hacer? Santiago, Chile: Edicio- 
nes Universidad Católica, Segunda Edición 2018: 35365.

28. Ley 20.606. Ministerio de Salud; Subsecretaría de Salud Pública. Sobre composición nutricional de los alimentos y su publicidad. Biblioteca del Congreso Nacional de Chile. Disponible en: https://www.leychile.cl/Consulta/ listaresultadosimple?cadena=20606 (Consultado el 30 enero de 2019).

29. Ruopeng A. Effectiveness of subsidies in promoting healthy food purchases and consumption: a review of field experiments. Public Health Nutr 2012; 16: 1215-28.

30. Hawkes C, Smith TG, Jewell J, Wardle J, Hammond R A, Sharon F, et al. Smart food policies for obesity prevention. Lancet 2015, 385: 2410-21. 